\title{
Recent Advancement in Hybrid Big Data Processing
}

\author{
Shuai Liu ${ }^{1,2} \cdot$ Huiyu Zhou ${ }^{3} \cdot$ Xiaochun Cheng ${ }^{4}$ \\ Published online: 14 December 2019 \\ (C) Springer Science+Business Media, LLC, part of Springer Nature 2019
}

\section{Editorial}

With rapid increment of communication technology, such as $5 \mathrm{G}$ etc., huge sensor based information has occupied our life. This kind of information is more hybrid with huge amount than traditional information, which needs new methodologies and computational architectures to process. Meantime, the huge information is changing current research domains.

Today, there are some novel computational architectures for huge information processing, for example, all deep learning related neural networks are novel architectures for huge information recognition with complex structure and characteristics. Fog computing, as well as edge computing become the main processing architectures for huge sensor based information because they are more effective than traditional parallel and cloud computational architectures. Also, security and privacy protection of the architectures are important issues to study.

Moreover, the computational methodologies are also evolved due to the hybrid types and structures of the information. Currently, information type does not only contain traditional information such as signals, images, texts, etc., but also contains SDMA, big multimedia, complex dirty data, etc. All these kinds of information needs novel computational methodologies to compress, classify and recognize, of course, with an acceptable computational complexity.

In this way, it is the right time to provide a platform to show the effective thoughts and methods to solve problems which are brought by hybrid big data. In particular, the uses of sophisticated and robust mathematical methods are also

Shuai Liu

cs.liu.shuai@gmail.com

1 Hunan Provincial Key Laboratory of Intelligent Computing and Language Information Processing, Hunan Normal University, Changsha, China

2 College of Information Science and Engineering, Hunan Normal University, Hohhot, China

3 School of Computing Science, University of Leicester, Leicester, UK

4 School of Computing Science, Middlesex University, London, UK

important in hybrid big data processing. Meantime, emerging methods which improve the efficiency of this domain are also encouraged in this issue.

So, this theme issue is proposed to provide an opportunity for researchers to publish their gifted theoretical and technological studies of advanced method in hybrid big data processing, and their novel engineering applications within this domain. In this theme issue, 12 in 33 submissions are accepted with accepted ratio $36.4 \%$.

The first article, titled "A Hybrid Task Scheduling Algorithm Based on Task Clustering", authored by Lei Chen from Georgia Southern University, USA, proposed a hybrid task scheduling algorithm based on task clustering HTSTC algorithm for heterogeneous multi-core processors [1]. Since problem of task communication overhead being higher than the task execution time had a direct negative impact on the makespan of task scheduling in the current scheduling algorithms, the proposed method used task clustering technology to integrate tasks that met the conditions into one cluster, and used task duplication method in the phase of processor selection. The algorithm effectively reduced the task communication overhead, and advanced the start time of the successor tasks. In the layering and task priority calculation phase, HTSTC took into account both the task communication overhead and task execution cost on different processors. The proposed algorithm effectively shortened the makespan of task scheduling. Experiments showed that HTSTC had superior performance when compared to HEFT and CPOP, two of the currently leading algorithms.

The second article titled "A Communication Method between High-speed UUV and Distributed Intelligent Nodes", authored by Yun Lin from Key Laboratory of Marine Information Acquisition and Security of Ministry of Industry and Information Technology of China, China, studied a Doppler distortion compensation method for underwater transmission of OFDM (Orthogonal Frequency Division Multiplexing) signals, which was used to realize real-time underwater acoustic communication between high-speed UUV (Unmanned Underwater Vehicle) and intelligent nodes [2]. The proposed method achieved excellent transmission performance with low computational complexity, and 
increased robustness to fast channel change with little priori information of the channel. This was because the proposed method used the TFFT (Taylor Fast Fourier Transformation) demodulation method in the underwater acoustic channel with severe Doppler shift. The feasibility and effectiveness of the proposed method were verified from recent UMA experiments, which proved that the method proposed in this paper provided an effective method for high-speed underwater acoustic communication.

The third article titled "Property of Self-similarity between Baseband and Modulated Signals", authored by J. A. Tenreiro Machado from Institute of Engineering, Polytechnic of Porto, Portugal, studied fractal characteristics of communication signals [3]. The proposed paper studied relation of self-similarity (SS) between baseband and modulated signals with the proof of their fractal relations. The authors considered modulated signal data of a certain time interval as a discrete timerandom sequence, and then proved that the ratio of physical quantities for the corresponding points was a constant at a corresponding time. They first proved that 5 fundamental modulated signals had SS on the premise that their baseband signals also had SS, and then verified the results by calculating the Hurst indices of these signals. Experiment of analysis and extraction of individual signal fingerprint features in communication radiation source was constructed to show that the proposed property SS was effective in identification and classification of communication radiation sources. The proposed research had broad application prospects, including electronic countermeasures, artificial intelligence and network security.

The forth article titled "Novel Temporal Perturbation-based Privacy-preserving Mechanism for Smart Meters", authored by Zhenquan Xu from State Key Laboratory of Information Engineering in Surveying, Mapping and Remote Sensing, China, proposed a temporal perturbation-based privacy-preserving mechanism to achieve a balance between privacy security and data availability for smart meters [4]. The proposed method solved problems of current privacy protection methods for smart meters, which did not efficiently protect users' privacy in terms of power consumption mode. The time disturbance model in this paper improved privacy security by staggering the acquisition and released time of smart meter data, destructed the load characteristics hidden in the power waveform, and realized the fuzzification of real consumption events. With the proposed method, the relative error of a single customer was maintained error range below $0.1 \%$, and the multi-user data aggregation error stabilized within $10 \%$. Evaluation results show that the proposed scheme performs well in protecting users' consumption pattern and is resistant to attacks by load detection; it also ensures data availability when used for intelligent services.

The fifth article titled "Radar Signal Recognition Based on Transfer Learning And Feature Fusion", authored by Lipeng Gao from Harbin Engineering University, China, designed a system for the automatic recognition of radar waveforms, which mainly used the obvious difference in Choi-Williams distribution (CWD) images of different modulated signals [5]. The proposed method transformed the problem of abstract radar signal intra-pulse recognition into one of image classification and optimized the superior performance of depth neural network in the field of image classification. In order to address the problem that the deep CNN was easy to over fit on a smallscale datasets, this study introduced transfer learning to finetune the pre-training network to adapt to this task. In experiment, a database containing 1800 images and 8 types of radar signal CWD images was established to verify the proposed method. Experimental results indicated that the trained CNNs had automatically ability to extract CWD image features, which solves the difficulties attributed to man-made features. The recognition accuracy with different SNR indicated that the proposed method recognized LPI radar signal feasibly under low-SNR conditions.

The sixth article titled "A Multi-Agent Simulation Method of Urban Land Layout Structure Based on FPGA", authored by Weina Fu from Hunan Normal University, China, provided a multi-agent simulation method based on FPGA for urban land layout [6]. The proposed method was used to improve the unavoidable birth defects of current methods, which led to incorrect reflection of evolution rules of urban land layout in simulation results. In this paper, The evolution rule of urban eco-land was explored by combination of cellular automata, dynamic reconfiguration and multi-agent methods. Random factors were added to make calculation results more in line with actual situation and reflect uncertainty of urban system. Example analysis results showed that simulation accuracy of the land layout structure of proposed method reached 92.4\%, which had high simulation accuracy. Moreover, conclusion was drawn that speed of urban expansion in the study area from 2007 to 2029 had gradually slowed down, and urban land-use pattern had changed from epitaxial expansion to intensive land-use. This was proved that the proposed method provided an effective reference for urban planning decisionmaking.

Coupled with the latest technologies on material science, biology and medicine, the OCS helped to realize operative control of objects of different natures. The seventh article titled "Analysis of the Mathematical Model Of Open Coaxial Measuring Microwave Converters", authored by Alexander Panchenko from Kharkiv National University of Radio Electronics, Ukraine, put forward a mathematical expression of middle-field OCS with a sample located outside the sensor that enabled allocation of basic elements to solve the electrodynamics problem in the OCS design [7]. The expression made it possible to shorten the computing time and reduce the number of approximations and heuristic assumptions, thereby improving the accuracy and adequacy of the results. The proposed method was used into OCS with different forms 
of working area and reached high effectiveness. The proposed method in this paper helped to develop algorithms and programs for qualitative analysis on OCS features, and to compute the parameters and functions of OCS design. In addition, the analysis method identified the basic and side effects of the qualitative level, and facilitate the construction of actual OCS designs.

Lower delay and higher throughput are the eternal study themes for some networks such as the mobile ad hoc networks (MANETs). The eighth article titled "The Application Study of Subgraph Isomorphism in the SDMA", authored by Tao Kai from 54th Research Institute of CETC, China, modeled a space time division multiple address for the MANET to meet the need for low delay of end-to-end data transmission in its particular applications [8]. The possibility of message transmission by the planning SDMA slot table decided if the pattern topology graph according to the SDMA slot table was included in the target topology graph. This paper solved this problem by employing a subgraph isomorphism model with a novel algorithm SGI(subgraph isomorphism) based on the Ullmann's simulation. The searching size of the proposed SGI algorithm was decreased effectively by the setting of the filtering strategies. The theory analyses and simulation showed that the computation complexity and the searching time of SGI were lower by comparing with Ullmann's algorithm, which denoted that the SGI algorithm had a wide application prospect for the space time division multiple address of MANET.

Nowadays, the uncertainties of covariance and unreasonable setting of expected threshold in cooperative tracking algorithm caused frequent switching of radar, and the existing sensor management was unsuited for the actual battlefield environment. The ninth article titled "Cooperative tracking technology of single target multi-sensor based on cooperative index", authored by Jianwen Gong from Naval Aviation University, China, studied this problem with single moving target by using a single maneuvering target multi-sensor cooperative tracking algorithm based on tracking accuracy partition and cooperative index optimization [9]. The expected threshold of cooperative tracking was revised by using covariance and information increment, the tracking accuracy was adaptively divided according to the battlefield situation, and the high-precision cooperative tracking of single target multisensor is realized by the co-index. Simulation results verified that it did not only solve the problem of frequent switching on and off of radar, but also improve the overall survivability of the system. Research results had significance to the increasingly fierce air combat of electronic countermeasures.

Internet of vehicles was a hot application in IoTs (internet of things) with urban transportation. Current adaptive torque balancing control of electric vehicle had shortcomings in electronic differential control of drive motor by using rotation speed mode. In order to solve this problem, the tenth article titled "Adaptive electronic differential control of vehicle by torque balance", authored by Sharon Wang from Loughborough University, UK, proposed an adaptive electronic differential control method of electric wheel-driven vehicle by torque balance [10]. The dynamics and kinematics of electric wheeled vehicle were analyzed by the principle of electronic differential speed under IoTs in this paper. The proposed method achieved the basic functions of differential speed and torque. Experimental results showed that the proposed method suppressed the abnormal jump interference factors of electronic differential control, as well as realized the differential functions in control system. It has far-reaching significance by providing a basic guarantee to realize adaptive electronic differential control system in electronic wheeldriven vehicle.

The eleven article titled "Human Behavior Understanding in Big Multimedia Data using CNN based Facial Expression Recognition", authored by Amin Ullah from Sejong University, Republic of Korea, analyzed human behavior by using facial expressions training with some famous TV-series videos [11]. The authors firstly detected faces using Violajones algorithm followed by tracking through KanadeLucas-Tomasi (KLT) algorithm. With HOG features utilized with SVM to classify faces of different actors, facial expressions was recognized using the proposed light-weight convolutional neural network $(\mathrm{CNN})$, particularly trained over KDEF dataset with extensive level of data augmentation. In order to achieve precise predictions of the behavior of individuals, the obtained results of FER with facial recognition in an occurrence matrix were integrated. Data augmentation techniques was used to overcome the faces from different views and lightening conditions in video data. The subjective and objective experimental evaluations prove better performance for both facial expression recognition and human behavior understanding.

Aiming at the problem that the frequency modulation slope obtained by the traditional linear frequency modulation (LFM) signal parameter estimation algorithm had high complexity with poor real-time performance and small SNR adaptation range, the twelve article titled "Research on Parameters Estimation of Signals Based on Fractal-Box Dimension", authored by Zhaoyue Zhang from Civil Aviation University, China, proposed an LFM signal parameter estimation algorithm based on the fractal-box dimension, and makes a detailed simulation analysis of the signal FM slope estimation [12]. The proposed method was utilized to evaluate the frequency modulation slope of the LFM signal, and the affect of signal amplitude and phase on the fractal-box dimension of the signal was taken into account. Compared with the traditional linear frequency modulation (LFM) signal parameter estimation algorithms, the anti-noise performance of the proposed algorithm was stronger, and the proposed algorithm was relatively simple and had good application value. The 
proposed method was a simple solution with good anti-noise and real-time performance, which had a fairly wide application value in engineering practice.

Acknowledgements The guest editors are thankful to our reviewers for their effort in reviewing these manuscripts. We also thank the Edit-inChief, Dr. Imrich Chlamtac for his supportive guidance during the entire process. The special issue is sponsored by Programs of National Natural Science Foundation of China under Grant 61502254, Program for Yong Talents of Science and Technology in Universities of Inner Mongolia Autonomous Region under Grant NJYT-18-B10, in part by the Open Project Program of the State Key Lab of CAD\&CG under Grant A1926, Zhejiang University, in part by the Hunan Provincial Science \& Technology Project Foundation (2018TP1018, 2018RS3065).

Publisher's Note Springer Nature remains neutral with regard to jurisdictional claims in published maps and institutional affiliations.

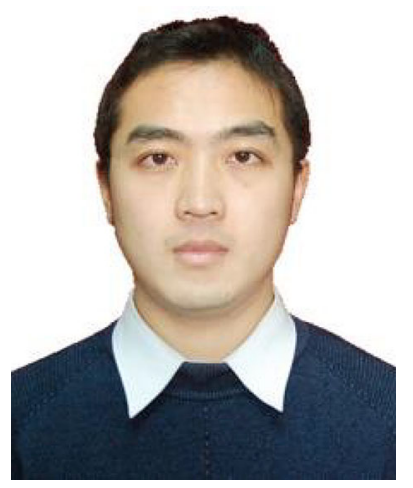

Shuai Liu received his bachelor's (2002), master's (2004) and doctoral (2011) degrees from the College of Computer Science and Technology, Jilin University. He now works as an Professor in the College of Information Science and Engineering, Hunan Normal University. He has published more than 30 papers in Elsevier, Springer, Oxford and other publishers. He is now interested in image processing, computer vision and fractals. He now acts as an (associate/guest) editor in IEEE Access, Mobile Networks and Application and so on. He also acts as a reviewer of IEEE Transactions on Fuzzy Systems, IEEE Transactions on Image Processing, Information Sciences, Applied Mathematics and Computation, IEEE Acces, and so.

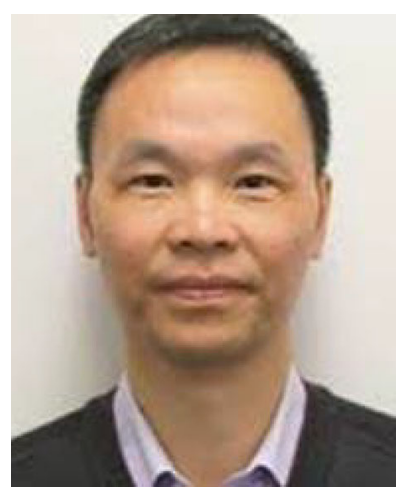

Huiyu Zhou, received a Bachelor of Engineering degree from Huazhong University of Science and Technology of China and a Master of Science degree from University of Dundee of United Kingdom, a doctor of Philosophy degree from HeriotWatt University, Edinburgh, United Kingdom. He currently is leading the Biomedical Image Processing Lab at University of Leicester. Dr. Zhou was the recipient of "CVIU 2012 Most Cited Paper Award". He currently serves as the Editor-in-Chief of Recent Advances in Electrical \& Electronic Engineering, Associate Editor of IEEE Transactions on Human-Machine Systems and IEEE Access, Editorial Board Member and Guest Editor of several refereed journals. His research has been or is supported by UK EPSRC, MRC, EU ICT, Royal Society, Innovate UK, Leverhulme Trust, Invest NI, Puffin Trust, Alzheimer Research (UK) and industry.

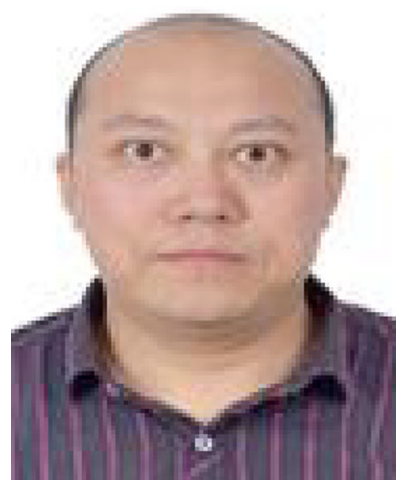

Xiaochun Cheng received the B.Sc degree in Computer Engineering in $1992, \mathrm{PhD}$ in Computer Science in 1996 , MBA in 2011. He has been EU Project Coordinator at Department of Computer Science in Middlesex University since 2012. He has contributed computing solutions to following EU Projects: Warehousing images in the digital hospital (WIDTH): interpretation, infrastructure, and integration (2011-2014) funded by EU FP7 Programme with Grant agreement number PIRSES-GA-2010-269,124 and Visual analytics for sense-making and analytic reasoning in criminal intelligence analysis (VALCRI) (2015-2018) funded through EU FP7 Programme with contract Number FP7-IP-608142. 